\title{
Formation and Evolution of Massive Black Holes in Star Clusters
}

\author{
Holger Baumgardt \\ Institute of Advanced Physical and Chemical Research RIKEN, 2-1 \\ Hirosawa, Wako-shi, Saitama 351-019, Japan \\ Junichiro Makino \\ Department of Astronomy, School of Science, The University of Tokyo, \\ 7-3-1 Hongo, Bunkyo-ku, Tokyo 113-0033, Japan
}

\section{Simon Portegies Zwart}

\begin{abstract}
Astronomical Institute "Anton Pannekoek" and Section Computational Science, University of Amsterdam, Kruislaan 403, 1098 SH Amsterdam, The Netherlands
\end{abstract}

\begin{abstract}
We present results of $N$-body simulations on the formation of massive black holes by run-away merging in young star clusters and the later dynamical evolution of star clusters containing massive black holes. We determine the initial conditions necessary for run-away merging to form a massive black hole and study the equilibrium profile that is established in the cluster center as a result of the interaction of stars with the central black hole. Our results show that star clusters which contain black holes have projected luminosity profiles that can be fitted by standard King models. The presence of massive black holes in (post-)core collapse clusters is therefore ruled out by our simulations.
\end{abstract}

\section{Introduction}

It has long been suspected that black holes with masses of a few hundred to a few thousand solar masses exist in the centers of some star clusters. Nevertheless observational evidence for their presence has been slow to accumulate. One of the best candidates to harbor a black hole is MGG-11, a young star cluster in the star-burst galaxy M82. Matsumoto et al. (2001) and Kaaret et al. (2001) have performed X-ray imaging of the central $\mathrm{kpc}$ of M82 and found an ultraluminous X-ray source with a luminosity that corresponds to a mass of more than $700 M_{\odot}$ if the object is accreting at the Eddington rate. Optical follow-up observations have shown that the position of the X-ray source coincides with that of MGG-11. McCrady et al. (2003) have obtained surface photometry and spectroscopy for a sample of star clusters in M82 and found that MGG-11 is deficient in low-mass stars. In addition, the cluster also had the smallest halfmass radius of all clusters in their study. Both findings imply that MGG-11 has a short relaxation time, so it seems possible that runaway collision of the most massive cluster stars could have formed a black hole. 


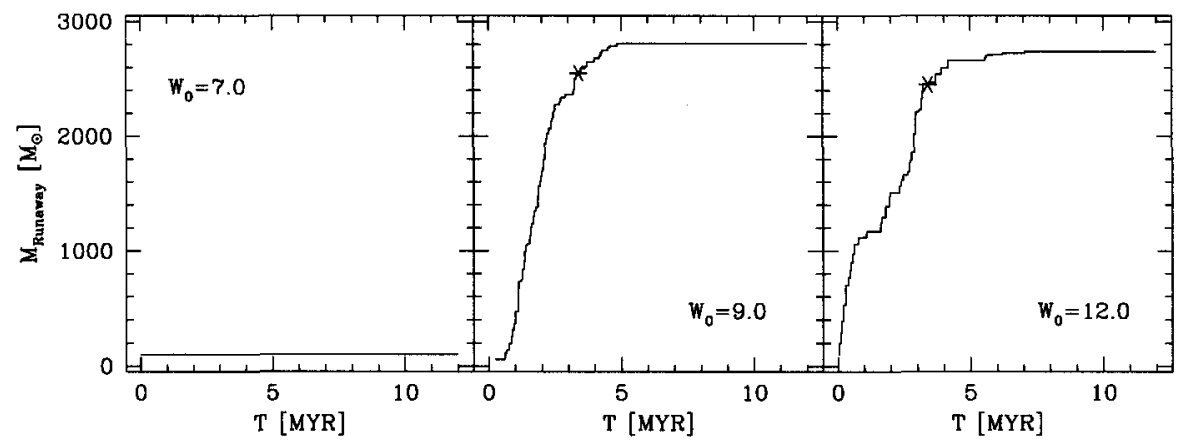

Figure 1. Mass of the most massive star for three clusters starting from King models with different central concentrations. Clusters with central concentrations $W_{0} \geq 9.0$ go into core-collapse quickly enough so that runaway merging leads to the formation of a star with several thousand solar masses. Asterisks mark the time of collapse of the runaway star to a black hole.

\section{Simulations of run-away merging}

In order to test if run-away merging can happen in MGG-11, we simulated the cluster evolution by $N$-body simulations. Our clusters contained $N=131.072$ stars which initially followed a Salpeter IMF between 1.0 and $100 M_{\odot}$. This leads to a total cluster mass and mass-to-light ratio which is in agreement with the observations. Since the density profile is not well known, we simulated clusters starting from King profiles with central concentrations in the range $7.0 \leq W_{0} \leq$ 12.0. The simulations were run for $T=12$ Myrs, which is compatible with the age of MGG-11 as found by McCrady et al. (2003). The initial half-mass radii of our clusters were adjusted such to match the observed cluster radius $r_{h p}=1.2$ pc after $T=12$ Myrs.

Fig. 1 shows the mass of the most massive star as a function of time for three different clusters. For a $W_{0}=7.0 \mathrm{King}$ model, the merger rate remains low throughout the simulation since the central density is low initially and the core collapse time is longer than the lifetime of the most massive cluster stars (about 3 Myrs). Hence the cluster starts expanding due to stellar evolution mass-loss before it can go into core-collapse and no run-away merging occurs.

King $W_{0}=9.0$ and $W_{0}=12.0$ models go into core-collapse quickly enough to concentrate a large fraction of their heavy mass stars in the center. Due to their large radii and masses collisions happen predominantly between the massive stars and lead to the formation of a super-massive star with a mass of more than $2000 M_{\odot}$ before going supernova. After the star is turned into a black hole, the merging activity declines. We conclude that an intermediate-mass black hole could have formed in MGG-11 if the initial cluster concentration was larger than $W_{0} \approx 9.0$. Since this is a typical concentration for globular clusters, it seems entirely possible that an intermediate mass black hole has formed in MGG-11. 


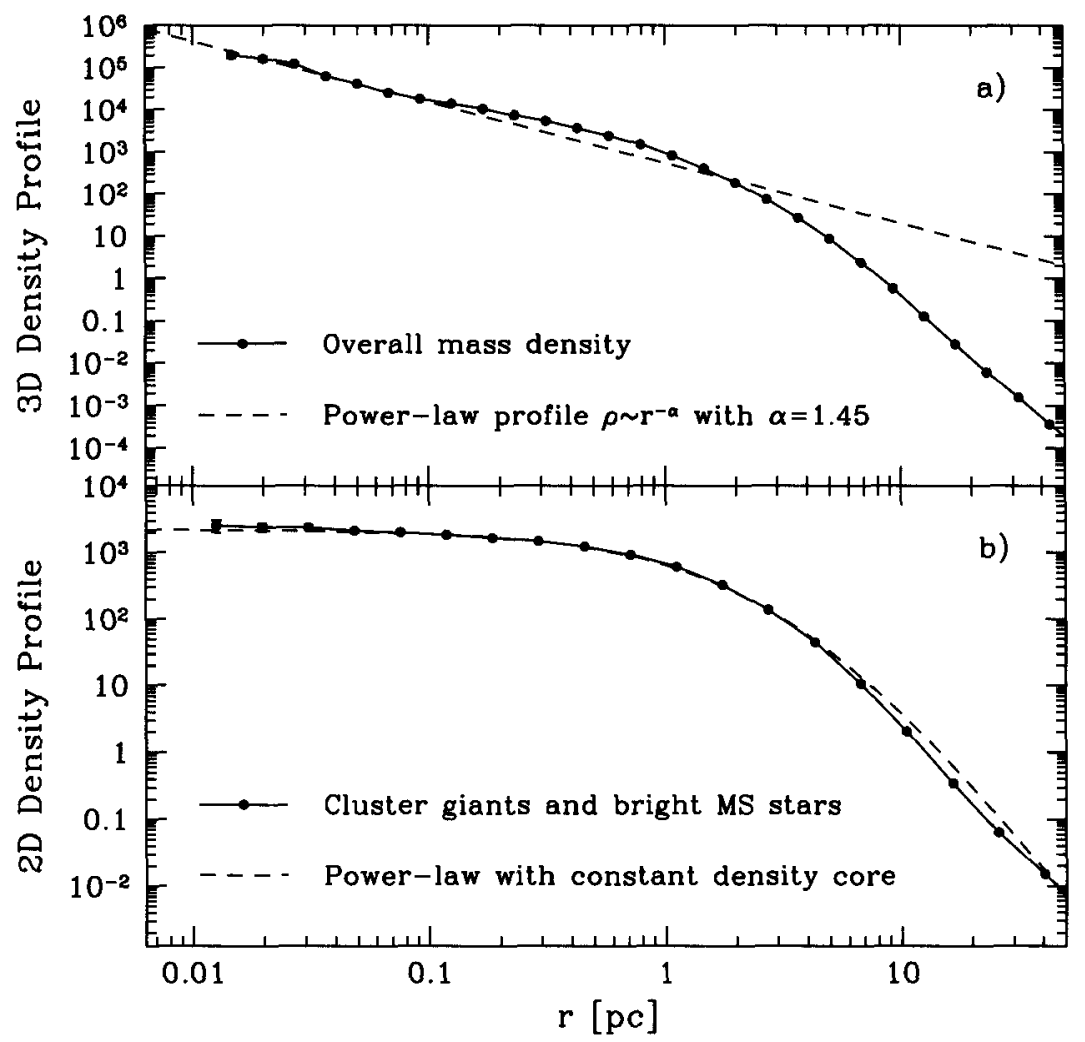

Figure 2. Density profile after $\mathrm{T}=12$ Gyrs of a star cluster containing $N=$ 131.072 stars and a central massive black hole of $1000 M_{\odot}$. In the center, a power-law profile with slope $\alpha=1.45$ is established in three dimensions (Fig. 2a). This leads to an almost flat luminosity profile in projection (Fig. 2b).

\section{Dynamical evolution of star clusters with massive central black holes}

After its formation, a massive black hole is likely to stay in the center of its parent cluster because it is too heavy to be expelled through close three-body interactions with other cluster stars. One might therefore expect that some galactic globular clusters contain massive black holes. Possible ways to detect them include the emission of gravitational waves if the black hole is in a tight binary with another black hole or neutron star, or the comparison of the central light and velocity profile with kinematical models. In order to study how star clusters with massive black holes evolve, we simulated the evolution of multimass clusters containing a $1000 M_{\odot}$ black hole in their center. The studied clusters started with a Kroupa (2001) IMF between 0.1 and $30 M_{\odot}$ and contained between 16.384 and 131.072 cluster stars. The simulations were performed for a duration of 12 Gyrs. Fig. 2 shows the final density profile for the cluster which started with $N=131.072$ stars. 
In three dimensions, the central density profile follows a power-law distribution $\rho \sim r^{-\alpha}$ with slope $\alpha=1.45$. This is slightly flatter than the results obtained from Fokker-Plank and Monte Carlo simulations: $\alpha=1.75$ (Bahcall \& Wolf 1976, Cohn \& Kulsrud 1978). One reason is that in the Fokker-Plank and Monte Carlo simulations the black hole was assumed to be at rest in the cluster center while in a real globular cluster it moves around in the center as a result of encounters with passing unbound stars. This leads to a flattening of the central profile. The projected distribution of bright stars is shown in Fig. $2 b$. It can be seen that the bright stars have a more or less constant density core. A cluster with a massive black hole in its center would therefore appear as a standard King profile cluster to an observer. We obtain the same results for clusters with other particle numbers.

\section{Conclusions}

We have performed simulations of the formation of massive black holes in star clusters and the later dynamical evolution of such systems. The ultra-luminous X-ray source in M82 might be an intermediate mass black hole formed through runaway merging of stars in a star cluster during the first 3 million years after cluster formation. We expect that star clusters with massive black holes will develop power-law cusps with relatively shallow slopes. It seems unlikely that massive black holes can be present in core-collapse clusters like M15, since in such clusters the central luminosity profile increases too steeply. Clusters with high central densities which appear to have a near constant density core might however be good places to look for massive black holes. A more detailed discussion of our results will be published in forthcoming papers (Portegies Zwart et al. 2003, Baumgardt et al. 2003).

\section{References}

Bahcall, J. N. \& Wolf, R. A. 1976, ApJ, 209, 214

Baumgardt, H., et al. 2003, in preparation

Cohn, H. \& Kulsrud, R. M. 1978, ApJ, 226, 1087

Kaaret, P., et al. 2001, MNRAS, 321, L29

Kroupa P., 2001, MNRAS, 322, 231

Matsumoto, H., et al. 2001, ApJ, 547, L25

McCrady, N., Gilbert, A. M., \& Graham, J. R. 2003, ApJ, 596, 240 (astro-ph/0306373)

Portegies Zwart, S., et al. 2003, ApJ, 593, 352 